\title{
OS AXIOMAS DE HUZITA-HATORI E O ENSINO DA GEOMETRIA EUCLIDIANA PLANA ATRAVÉS DA CONSTRUÇÃO DE POLÍGONOS
}

\author{
Odair José Pin, Eugenia Brunilda Opazo Uribe \\ Universidade Federal do Mato Grosso do Sul, PET Conexões de Saberes - Matemática, Três Lagoas, MS. E- \\ mail: odairjosepin@gmail.com \\ Agência de fomento: Programa de Educação Tutorial
}

\begin{abstract}
RESUMO
O trabalho apresenta resultados de uma atividade de pesquisa de um aluno do Curso de Matemática no âmbito do Programa de Educação Tutorial, que visa o estudo dos axiomas de Huzita-Hatori: axiomas por meio de dobraduras (origami); e como relacioná-los com o ensino da Geometria Euclidiana Plana, de forma útil, agradável e dinâmica para os alunos. A proposta é desenvolver uma atividade de revezamento, na qual cada aluno é responsável por uma parte da construção geométrica sugerida, desenvolvendo assim o conteúdo básico de geometria plana e o estudo de polígonos. O desenvolvimento do mesmo foi realizado através de levantamento bibliográfico, estudo teórico, discussões, apresentação de seminários, preparação e elaboração de uma oficina, e elaboração do relatório final. Conclui-se que a associação dos axiomas de Huzita Hatori com o básico da Geometria Euclidiana Plana é um método simbólico-cognitivo útil no aprendizado e na construção de polígonos, além de ser uma atividade diferenciada e divertida.
\end{abstract}

Palavras-chave: Axiomas, Huzita-Hatori, Geometria Euclidiana Plana, Dinâmica, Origami.

\section{HUZITA-HATORI AXIOMS AND TEACHING OF EUCLIDEAN GEOMETRY THROUGH THE CONSTRUCTION OF FLAT POLYGONS}

\begin{abstract}
This paper is the result of the research activity of a student in Mathematics Graduation in the Tutorial Educational Program, aimed at the study of Huzita-Hatori axioms: axioms by means of folding (origami); and how to relate them with the teaching of Euclidean geometry, so useful, dynamic and enjoyable for the students. The proposal is to develop a relay activity, in which each student is responsible for a part of geometric construction suggested developing so the basic content of plane geometry and the study of polygons. The development of it was accomplished through bibliographical survey, theoretical study, discussions, seminars, preparation and elaboration of a workshop, and preparing the final report. It is concluded that the Association of Huzita-Hatori axioms with the basics of Plane Euclidean Geometry is a symbolic-cognitive method useful in learning and in the construction of polygons, as well as being a distinctive and entertaining activity.
\end{abstract}

Keywords: Axioms, Huzita-Hatori, Plane Euclidian Geometry, Dynamics, Origami. 


\section{INTRODUÇÃO}

A Geometria Euclidiana Plana é um tema bem complexo para o ensino, principalmente pelo fato de que não é algo facilmente manipulável, muito menos trabalhado de forma profunda e significativa no ensino atualmente. Vários fatores levam a essa defasagem, incluindo o desinteresse, a falta de criatividade e tempo, e o fato do professor se sentir desestimulado para se aprofundar nisso.

No entanto, ela não precisa ser mostrada somente através de lousa e giz: existem métodos lúdicos e divertidos de ensinar geometria. Um deles é o origami, método criativo de ensinar e utilizar conceitos aprendidos em sala de aula para a construção de figuras. Como cita Dias (2015), em seu trabalho: "O uso do Origami como recurso didático-metodológico para o ensino de geometria"

[...] Por intermédio do uso das dobraduras de papel, o ensino da geometria passa a favorecer associações entre conteúdos abstratos e problemáticas cotidianas, isto porque na confecção de cada dobradura tornam-se extremamente necessárias articulações de estratégias geométricas para efetuar tais construções. Além disso, constituem-se em material didático que transforma o ensino-aprendizagem em uma atividade atraente e motivadora onde os alunos podem desenvolver sua experimentação geométrica e a visão espacial.(p. 11)

O presente trabalho está associado à atividade de pesquisa "Estudo sobre Geometria através de Jogos, Resolução de Problemas e do Origami" desenvolvida pelo grupo PET Conexões de Saberes - Matemática da UFMS, Campus de Três Lagoas.

O objetivo do presente trabalho é propor uma atividade diferenciada, para alunos de 70 e 8 o ano do ensino fundamental, com o origami, através da utilização dos axiomas de Huzita-Hatori na construção de polígonos. A proposta é desenvolver uma dinâmica de revezamento entre equipes, associando tais axiomas aos axiomas de Euclides na Geometria Plana, tornando a aula mais proveitosa e divertida.

\section{METODOLOGIA}

O desenvolvimento do trabalho foi realizado através de uma pesquisa bibliográfica, estudo teórico dos conceitos básicos da Geometria Plana, discussões em grupo, apresentação de seminários e resolução de exercícios, preparação e desenvolvimento de uma oficina, análise dos resultados e da avaliação da oficina para implementação de mudanças e adaptações, elaboração do relatório final.

\section{RESULTADOS}

Primeiramente, fez-se uma pesquisa detalhada dos axiomas, e como relacioná-los com a Geometria Euclidiana Plana. Humiaki Huzita, matemático ítalo-japonês e destaque no estudo do origami, descreveu seis operações básicas para a construção de figuras por meio de dobradura. Em 1989, Jaques Justin escreveu um artigo onde ele citava, além dos seis axiomas de Huzita, um outro axioma, descrito por Hoshiro Hatori. Dessa forma, surgem os sete axiomas de Huzita-Hatori, descritos abaixo:

- Axioma I: Dados dois pontos $\mathrm{P}_{1}$ e $\mathrm{P}_{2}$, existe uma única dobra que passa por esses dois pontos. 


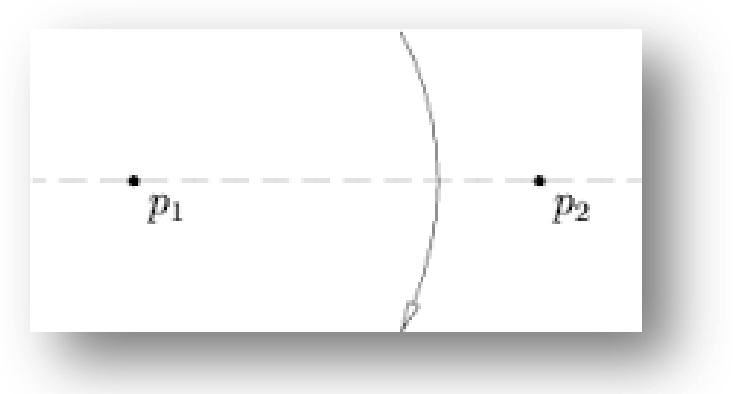

O axioma acima está associado a um dos axiomas da Geometria Euclidiana Plana, descrito Barbosa (2012), que diz: Dados dois pontos distintos, existe uma única reta que contém esses pontos.

- Axioma II: Dados dois pontos $\mathrm{P}_{1}$ e $\mathrm{P}_{2}$, existe uma única dobra que coloca $\mathrm{P}_{1}$ sobre $\mathrm{P}_{2}$.

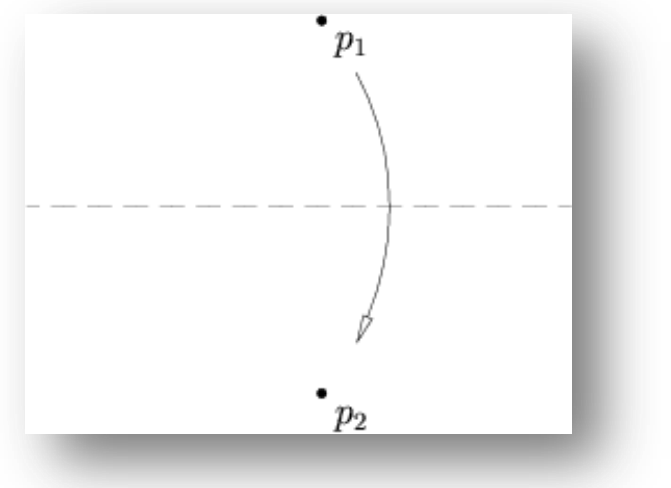

O axioma acima está associado à existência e unicidade da mediatriz do segmento $\mathrm{P}_{1} \mathrm{P}_{2}$. Perceba que, ao dobrar a folha, a dobra dividirá o segmento em duas partes, de mesma medida.

- Axioma III: Dadas duas retas $\mathrm{I}_{1}$ e $\mathrm{I}_{2}$, existe uma dobra que colocam $\mathrm{I}_{1}$ sobre $\mathrm{I}_{2}$.

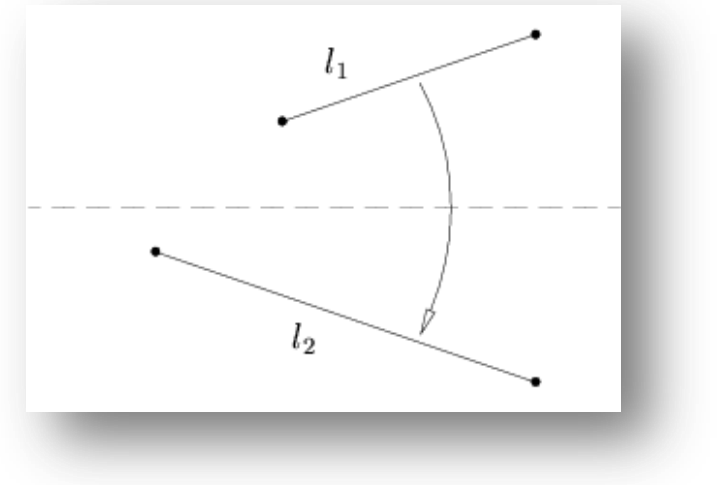

O axioma acima está associado às bissetrizes dos ângulos formados pela intersecção das duas retas. As retas que contêm essas bissetrizes são as dobras que fazem $I_{1}$ e $I_{2}$ coincidirem. Perceba que há três soluções diferentes: Existirem duas dobras, se elas se concorrem; existir uma somente, que é mediatriz de todos os segmentos formados de medida igual à distância entre essas retas, tomando um ponto de $I_{1}$ e outro de $I_{2}$ como extremos e $I_{1} / / I_{2}$; ou infinitas, levando em consideração $I_{1}=I_{2}$, e qualquer perpendicular a elas leva uma até a outra.

- Axioma IV: Dados um ponto $\mathrm{P}_{1}$ e uma reta $\mathrm{I}_{1}$, existe uma única dobra, perpendicular à $\mathrm{I}_{1}$, que passa por $\mathrm{P}_{1}$. 


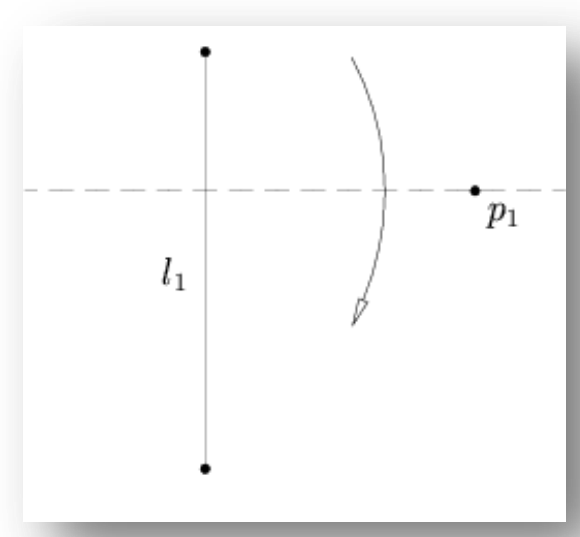

O axioma acima está associado à existência e unicidade da reta perpendicular que passa por um ponto.

- Axioma $V$ : Dados dois pontos $\mathrm{P}_{1}$ e $\mathrm{P}_{2}$ e uma reta $\mathrm{I}_{1}$ desde que a distância de $\mathrm{P}_{1}$ à $\mathrm{P}_{2}$ seja superior ou igual à distância de $P_{2}$ à $I_{1}$, existe uma dobra que leva $P_{1}$ até $I_{1}$ e passa por $P_{2}$.

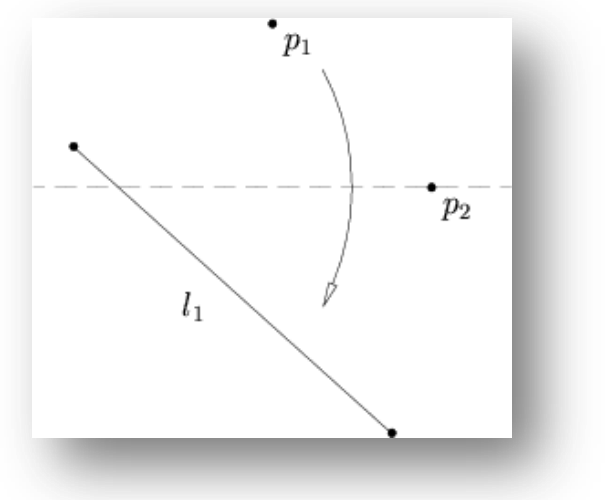

De acordo com Furuya (2009), a quantidade de soluções do Axioma 5 pode ser 0, 1 ou 2, dependendo da posição dos pontos e da reta, pois o problema é equivalente a encontrar a intersecção da reta $I_{1}$ com a circunferência de centro $P_{2}$ passando por $P_{1}$.

- Axioma VI: Dados dois pontos $\mathrm{P}_{1}$ e $\mathrm{P}_{2}$ e duas retas $\mathrm{I}_{1}$ e $\mathrm{I}_{2}$, desde que $\mathrm{I}_{1}$ e $\mathrm{I}_{2}$ não forem paralelas, existem dobras que levam $P_{1}$ até $I_{1}$ e $P_{2}$ até $I_{2}$.

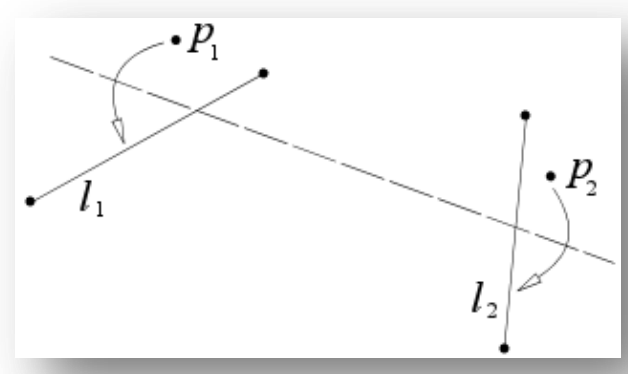

Após realizar a dobra, teremos as medianas dos segmentos onde $\mathrm{P}_{1} \mathrm{P}_{1}{ }^{\prime}$ e $\mathrm{P}_{2} \mathrm{P}_{2}{ }^{\prime}$, onde $\mathrm{P}_{1}{ }^{\prime}$ e $\mathrm{P}_{2}{ }^{\prime}$ são os pontos gerados em $\mathrm{I}_{1}$ e $\mathrm{I}_{2}$, respectivamente, após a dobra. Este axioma não é possível de se construir com régua e compasso.

- Axioma VII: Dados um ponto $\mathrm{P}$ e duas retas $\mathrm{I}_{1}$ e $\mathrm{I}_{2}$, existe uma dobra, perpendicular à $\mathrm{I}_{2}$, que leva $\mathrm{P}_{1}$ até $I_{1}$. 


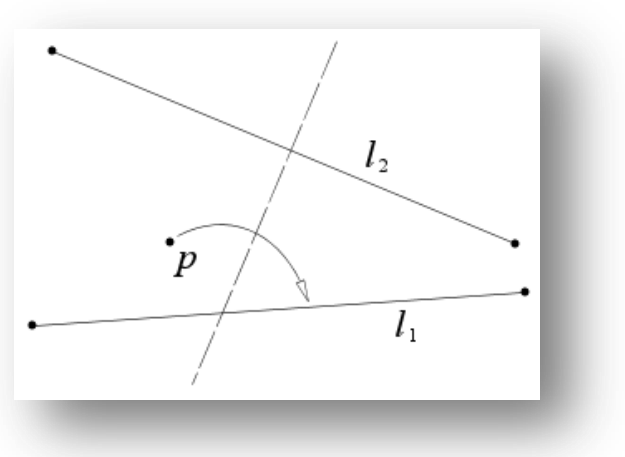

O último axioma é discutível, pois decorre das construções repetidas do Axioma $I V$ descrito anteriormente, nos seguintes passos:

- Construa uma dobra perpendicular à $I_{2}$, passando por $P$. Chame-a de $d_{1}$.

- Construa uma dobra perpendicular à $d_{1}$, de forma a levar $P$ até $I_{1}$. Chame-a de $d_{2} e$ marque a intersecção de $\mathrm{d}_{2}$ com l$_{1}$;

- Por último, construa outra perpendicular, em relação à $d_{2}$, passando pela intersecção dela com I.

Uma vez exploradas essas relações, a proposta é realizar uma atividade de revezamento entre os alunos, propondo problemas de construção geométrica e a construção de polígonos, regulares ou não, utilizando somente dobraduras ou utilizando régua e compasso para verificação dos axiomas.

Como forma de experimentação, foi realizada uma oficina com os graduandos do curso de Licenciatura em Matemática da Universidade Federal do Mato Grosso do Sul, Campus de Três Lagoas. A oficina foi separada em dois momentos. No primeiro momento, explorou-se a construção, verificação dos axiomas e identificação dos mesmos. Logo após, sugeriu-se aos alunos a construção de triângulos, quadrados, retângulos, entre outros polígonos, visando a compreensão e utilização de determinadas propriedades dessas figuras e a aplicação dos axiomas em cada construção. Foi proposto também que os alunos pensassem em um método, somente por dobraduras, para provar que a soma dos ângulos internos de um triângulo é 180 graus. Como primeira oficina, ainda não foi proposta nessa parte o revezamento.

\section{DISCUSSÃO}

Os graduandos do curso de matemática contribuíram muito com a oficina, sendo receptivos e críticos. Na primeira parte da oficina, houve uma dificuldade maior de compreensão, até porque alguns axiomas são difíceis de visualizar e construir. Alguns deles não são nem possíveis de serem construídos por métodos convencionais, como o Axioma VI, o que dificulta a associação dos conteúdos. Isso foi bastante positivo e construtivo para a proposta, e a enriqueceu ainda mais. Na segunda parte, as atividades propostas renderam mais, considerando que os participantes já tinham certa facilidade em origami e conheciam as propriedades dos polígonos. No geral, a oficina obteve resultados satisfatórios.

\section{CONCLUSÃO}

Conclui-se que a associação dos axiomas de Huzita - Hatori com o básico da Geometria Euclidiana Plana é um método simbólico-cognitivo útil no aprendizado e na construção de polígonos, além de ser uma atividade diferenciada e divertida. Além disso, a pesquisa e a oficina contribuíram muito para a formação profissional de um futuro professor, estimulando minha criatividade e me proporcionando experiência. Futuramente, será dado continuidade a este 
trabalho com a construção de um jogo que utilize do revezamento para a construção de polígonos, o que, na visão dos autores, é de grande importância para estimular o trabalho em equipe.

\section{REFERÊNCIAS}

Barbosa, João Lucas Marques. Geometria Euclidiana Plana. 11ํe ed. Coleção SBM. 2012.

Cavacami, E.; Furuya, Y. K. S. Explorando geometria com origami. 2009. Disponível no Departamento de Matemática da UFSCAR: http://www.dm.ufscar.br/ yolanda/origami/origami.pdf

Dias, M. C. de O. O uso do origami como recurso didático-metodológico para o ensino de geometria. 2015. Disponível no Repositório Institucional da UFJF: https://repositorio.ufjf.br/jspui/handle/ufjf/1443 